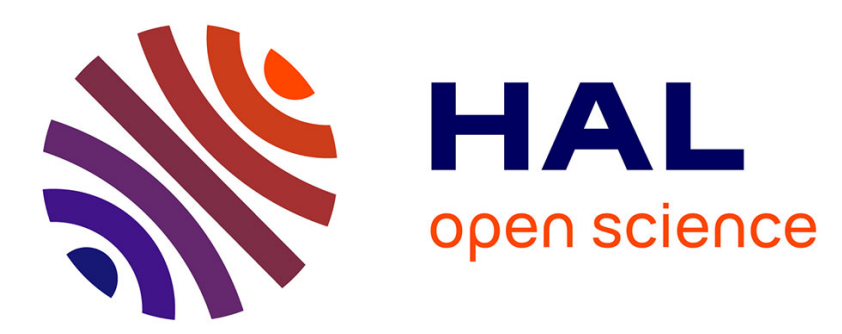

\title{
Recursive identification for Hammerstein systems with bounded noise
}

\author{
Mathieu Pouliquen, Eric Pigeon, Olivier Gehan
}

\section{To cite this version:}

Mathieu Pouliquen, Eric Pigeon, Olivier Gehan. Recursive identification for Hammerstein systems with bounded noise. 52nd IEEE Conference on Decision and Control, Dec 2013, Firenze, Italy. pp.4919-4924, 10.1109/CDC.2013.6760661 . hal-00965067

\section{HAL Id: hal-00965067 https://hal.science/hal-00965067}

Submitted on 25 Mar 2014

HAL is a multi-disciplinary open access archive for the deposit and dissemination of scientific research documents, whether they are published or not. The documents may come from teaching and research institutions in France or abroad, or from public or private research centers.
L'archive ouverte pluridisciplinaire HAL, est destinée au dépôt et à la diffusion de documents scientifiques de niveau recherche, publiés ou non, émanant des établissements d'enseignement et de recherche français ou étrangers, des laboratoires publics ou privés. 


\title{
Recursive Identification for Hammerstein Systems with Bounded Noise
}

\author{
Mathieu Pouliquen, Eric Pigeon, Olivier Gehan \\ Control Group, GREYC CNRS UMR 6072 \\ ENSICAEN, 06 Bd du Marechal Juin \\ 14050 Caen Cedex, France \\ mathieu.pouliquendunicaen. fr
}

\begin{abstract}
In this paper, we consider the identification of Hammerstein systems in presence of bounded disturbances. The proposed identification algorithm is a recursive algorithm. It ensures that the estimation error is bounded and allows an online separation of the nonlinear part and linear part. Furthermore, some stability and convergence results are demonstrated and a simulation example is given to illustrate the performances of the presented method.
\end{abstract}

\section{INTRODUCTION}

The Hammerstein systems are the nonlinear systems consisting of a static nonlinearity block followed by a linear dynamic system. The identification of such systems has received much interest for the last decades ([22], [8], [16], [15], [2], [3], [7], [10], [27], [14], [28], [4], [13], [18], [17] and the four sessions devoted to block oriented nonlinear identification at SYSID 2012). The reason is that these systems take into account nonlinearities commonly encountered in practice, generated by the technological limitations necessary for the proper functioning of the system (saturation, the limit stops, etc.).

For Hammerstein systems or not, all practical identification algorithms have to deal with measurements corrupted by noise and most of the previous works consider the stochastic noise assumption (except [7] discussed later). In many cases (unknown probability distribution of the disturbances, modeling inaccuracy) such assumption cannot be done and the bounded noise assumption seems most appropriate. To address this identification problem we will use in this paper Set Membership Identification (SMI) algorithms (see [21], [20], [26], [12] and [1]) and particularly the Optimal Bounding Ellipsoid (OBE) algorithms. These algorithms have a low computational complexity and they are appropriated to handle the identification problem in presence of bounded disturbances. Their principle consists in the estimation of a feasible set of parameters which must be consistent with the measurement data and the model structure. Some important contributions have been presented in [11], [9], [6] and [25].

The aim of this paper is to propose such recursive identification algorithm for Hammerstein systems subjected to bounded noises. The proposed algorithm is a two stages algorithm: the first stage updates a parameters vector from the new measurements, the second stage separates recursively the linear and nonlinear part. This separation induces an internal disturbance term and a great difficulty is to demonstrate the stability of the algorithm even with this internal disturbance term. This is one of the main contributions of this paper.

Briefly, the paper is organized as follows: section II introduces the model structure and states the objective of the study. In section III, the adaptation algorithm is proposed and some stability and convergence properties are addressed. Some simulation results are given in section IV. Finally, section $\mathrm{V}$ concludes the paper. Appendices contain most of the proofs.

\section{PROBLEM STATEMENT}

The Hammerstein system presented in figure 1 is described by

$$
A(q) y_{t}=q^{-d} B(q) x_{t}+v_{t}
$$

with

$$
\left\{\begin{array}{l}
B(q)=b_{0}+b_{1} q^{-1}+\cdots+b_{n_{b}} q^{-n_{b}} \\
A(q)=1+a_{1} q^{-1}+\cdots+a_{n_{a}} q^{-n_{a}}
\end{array}\right.
$$

and

$$
x_{t}=h\left(u_{t}\right)
$$

where $u_{t}$ and $y_{t}$ are respectively the system input and the system output. $x_{t}$ is the internal signal and $v_{t}$ is an unknown but bounded disturbing term (noise measurement, state disturbances, modeling inaccuracy, etc.).

$h($.$) is a static nonlinear function without memory. It is$ represented by a sum of the known nonlinear functions $h_{1}($.$) ,$ $h_{2}(),. \ldots, h_{n_{h}}($.$) :$

$$
h\left(u_{t}\right)=\mu_{1} h_{1}\left(u_{t}\right)+\mu_{2} h_{2}\left(u_{t}\right)+\cdots+\mu_{n_{h}} h_{n_{h}}\left(u_{t}\right)
$$

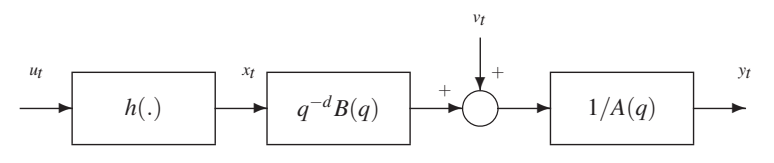

Fig. 1. Studied Hammerstein system

The objective: Given the delay $d$, the degrees $n_{a}, n_{b}$ and $n_{h}$, estimate recursively the parameters $\left\{a_{i}\right\},\left\{b_{i}\right\}$ and $\left\{\mu_{i}\right\}$ of the model based on the input-output measurements of the system described by (1), (2) and (3). 
Remark 1: In [7] a similar problem is treated. The difference lies in the fact that in [7] the linear part is a $\mathrm{OE}$ model, the algorithm is not a recursive algorithm, several experiments are needed to identify some points of $h($.$) and$ the separation of the linear and nonlinear part is not realized in the same time.

In this paper, so as to be able to identify the parameters, some assumptions are needed:

A1 The delay $d$, the orders of the linear part and of the nonlinear part $\left(n_{a}, n_{b}\right.$ and $\left.n_{h}\right)$ are supposed to be available;

A2 The coefficient $b_{i}$ are such that $b_{0}=1$;

A3 Regardless of the disturbance nature, an upper bound is supposed to be available on the magnitude of $v_{t}$ :

$$
\left|v_{t}\right| \leq \delta_{v_{t}}
$$

Remark 2: The assumption $[A 2]$ is necessary so as to satisfy the identifiability condition. Without this assumption we are not able to distinguish the solution $G(q) h\left(u_{t}\right)$ from the solution $(a G(q))\left(\frac{1}{a} h\left(u_{t}\right)\right)$ with $a \neq 0$.

Remark 3: About [A3], it is not necessary to know exactly the value of $\delta_{v_{t}}$. When identifying a system for which this bound is unknown, it is possible to process by dichotomy to refine the results of the estimation.

\section{IDENTIFICATION ALGORITHM AND ANALYSIS}

From (1), (2) and (3) the output of the Hammerstein system is expressed as follows:

$$
y_{t}=\sum_{k=0}^{n_{b}} b_{k} \sum_{i=1}^{n_{h}} \mu_{i} h_{i}\left(u_{t-k-d}\right)-\sum_{k=1}^{n_{a}} a_{k} y_{t-k}+v_{t}
$$

By setting

$$
\theta^{*}=\left(\begin{array}{c}
\mu_{1} \\
\vdots \\
b_{n_{b}} \mu_{1} \\
\mu_{2} \\
\vdots \\
b_{n_{b}} \mu_{2} \\
\vdots \\
\mu_{n_{h}} \\
\vdots \\
b_{n_{b}} \mu_{n_{h}} \\
a_{1} \\
\vdots \\
a_{n_{a}}
\end{array}\right) \quad \phi_{t}=\left(\begin{array}{c}
h_{1}\left(u_{t-d}\right) \\
\vdots \\
h_{1}\left(u_{t-d-n_{b}}\right) \\
h_{2}\left(u_{t-d}\right) \\
\vdots \\
h_{2}\left(u_{t-d-n_{b}}\right) \\
\vdots \\
h_{n_{h}}\left(u_{t-d}\right) \\
\vdots \\
h_{n_{h}}\left(u_{t-d-n_{b}}\right) \\
-y_{t-1} \\
\vdots \\
-y_{t-n_{a}}
\end{array}\right)
$$

the system is rewritten in the compact form

$$
y_{t}=\phi_{t}^{T} \theta^{*}+v_{t}
$$

$\theta^{*} \in \mathbb{R}^{n}$ is the unknown parameters vector to be identified with $n=\left(1+n_{b}\right) n_{h}+n_{a}$ the number of parameters.

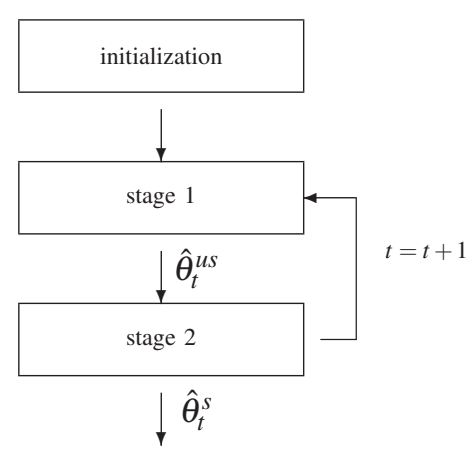

Fig. 2. A two stages algorithm

The first $\left(1+n_{b}\right) n_{h}$ terms of the vector $\theta^{*}$ can be inserted in a $\left(1+n_{b}\right) \times n_{h}$ matrix as follows

$$
M_{\theta^{*}}=\left(\begin{array}{ccc}
\theta^{*}(1) & \cdots & \theta^{*}\left(\left(1+n_{b}\right)\left(n_{h}-1\right)+1\right) \\
\vdots & & \vdots \\
\theta^{*}\left(1+n_{b}\right) & \cdots & \theta^{*}\left(\left(1+n_{b}\right) n_{h}\right)
\end{array}\right)
$$

which can be rewritten as

$$
M_{\theta^{*}}=\left(\begin{array}{c}
1 \\
b_{1} \\
\vdots \\
b_{n_{b}}
\end{array}\right)\left(\begin{array}{lll}
\mu_{1} & \cdots & \mu_{n_{h}}
\end{array}\right)
$$

Such structure will be used to separate the parameters $\left\{b_{i}\right\}$ and $\left\{\mu_{i}\right\}$. By now, our aim in what follows is to

- propose an estimation algorithm based on (6) and (4);

- estimate a parameters vector structured as (7);

- give a proof of stability and convergence of this algorithm.

The algorithm considered in this paper recursively estimates the parameters $\theta^{*}$. For this purpose the one-step-ahead output predictor is defined as follows ([19]):

$$
\hat{y}_{t}=y_{t}-v_{t}=\phi_{t}^{T} \theta^{*}
$$

Before introducing the parameter adaptation algorithm, let define the a priori and a posteriori predictors as

$$
\left\{\begin{array}{l}
\hat{y}_{t, \hat{\theta}_{t-1}}=\phi_{t}^{T} \hat{\theta}_{t-1} \\
\hat{y}_{t, \hat{\theta}_{t}}=\phi_{t}^{T} \hat{\theta}_{t}
\end{array}\right.
$$

$\hat{\theta}_{t}$ represents an estimation of the parameters vector at the actual time $t$. Let define also $\hat{\theta}_{t}^{s}$ and $\hat{\theta}_{t}^{u s}$ as follows:

- $\hat{\theta}_{t}^{s}$ is an estimation which satisfies (7);

- $\hat{\theta}_{t}^{u s}$ is an estimation which does not satisfy (7).

Subscripts $s$ and $u s$ respectively stand for "structured" and "un-structured". 


\section{A. The adaptation algorithm.}

To address the identification problem we will take inspiration from the OBE identification methods presented in [6], [25] and [24]. As depicted on Fig. 2, at the time $t$ the proposed algorithm is divided in two successive stages:

stage 1 Update of the estimated parameters vector taking into account the new observation vector $\phi_{t}$ and the new output $y_{t}$ : estimation of $\hat{\theta}_{t}^{u s}$ from $\hat{\theta}_{t-1}^{s}$;

stage 2 Structuring of the estimated solution: estimation of $\hat{\theta}_{t}^{s}$ from $\hat{\theta}_{t}^{u s}$.

The estimated parameters vector must satisfy (7) and has to maintain the prediction error below a bound defined from the upper bound on the disturbance $v_{t}$. Under some conditions describes below, the following estimation algorithm provides such estimation:

stage 1

$$
\left\{\begin{array}{l}
\hat{\theta}_{t}^{u s}=\hat{\theta}_{t-1}^{s}+\Gamma_{t} \tilde{y}_{t, \hat{\theta}_{t-1}^{s}} \\
\Gamma_{t}=\frac{P_{t-1}^{s} \phi_{t} \sigma_{t}}{\lambda+\phi_{t}^{T} P_{t-1}^{s} \phi_{t} \sigma_{t}} \\
\tilde{y}_{t, \hat{\theta}_{t-1}^{s}=y_{t}-\hat{y}_{t, \hat{\theta}_{t-1}^{s}}}\left(P_{t}^{u s}\right)^{-1}=\lambda\left(P_{t-1}^{s}\right)^{-1}+\phi_{t} \sigma_{t} \phi_{t}^{T}
\end{array}\right.
$$

stage 2

$$
\left\{\begin{aligned}
\hat{\theta}_{t}^{s} & =S\left(\hat{\theta}_{t}^{u s}\right) \\
P_{t}^{s} & =\frac{1}{1-\zeta} P_{t}^{u s}+\frac{1}{\zeta} W_{t}
\end{aligned}\right.
$$

Some useful insights on the algorithm are given below.

\section{about stage 1:}

$\hat{\theta}_{t-1}^{s}$ and $P_{t-1}^{s}$ are computed at the previous iteration.

$\tilde{y}_{t, \hat{\theta}_{t-1}^{s}}$ is the a priori prediction error, $0<\lambda \leq 1$ is the forgetting factor and $\sigma_{t}$ is a switching flag given by:

$$
\sigma_{t}=\left\{\begin{array}{l}
\frac{\lambda}{\phi_{t}^{T} P_{t-1}^{s} \phi_{t}}\left(\left|\frac{\tilde{y}_{t, \hat{\theta}_{t-1}^{s}}}{\delta_{t}}\right|-1\right) \\
\quad \text { if }\left(\left|\frac{\tilde{y}_{t,}, \hat{\theta}_{t-1}^{s}}{\delta_{t}}\right|>1\right) \text { and }\left(\phi_{t}^{T} P_{t-1}^{s} \phi_{t}>0\right) \\
0 \text { otherwise }
\end{array}\right.
$$

Its role is specified below.

From (8) the unstructured a posteriori prediction error $\tilde{y}_{t, \hat{\theta}_{t}^{u s}}=y_{t}-\hat{y}_{t, \hat{\theta}_{t}^{u s}}$ can be written as

$$
\tilde{y}_{t, \hat{\theta}_{t}^{u s}}=\left(1-\phi_{t}^{T} \Gamma_{t}\right) \tilde{y}_{t, \hat{\theta}_{t-1}^{s}}
$$

which can also be rewritten, by using the expression for $\Gamma_{t}$, as:

$$
\tilde{y}_{t, \hat{\theta}_{t}^{u s}}=\frac{\lambda}{\lambda+\phi_{t}^{T} P_{t-1}^{s} \phi_{t} \sigma_{t}} \tilde{y}_{t, \hat{\theta}_{t-1}^{s}}
$$

Using the value of $\sigma_{t}$ for $\sigma_{t} \neq 0$ (11) yields:

$$
\left|\frac{\tilde{y}_{t, \hat{\theta}_{t}^{u s}}}{\delta_{t}}\right|=1
$$

This clearly shows that, provided that $\phi_{t}^{T} P_{t-1} \phi_{t}>$ 0 , the introduction of $\sigma_{t}$ in the first part of the algorithm ensures the following property:

$$
\left\{\begin{array}{l}
\text { if }\left|\frac{\tilde{y}_{t, \hat{\theta}_{t-1}^{s}}}{\delta_{t}}\right|>1 \text { then } \sigma_{t} \neq 0 \text { and }\left|\frac{\tilde{y}_{t, \hat{\theta}_{t}^{u s}}}{\delta_{t}}\right|=1 \\
\text { if }\left|\frac{\tilde{y}_{t, \hat{\theta}_{t-1}^{s}}}{\delta_{t}}\right| \leq 1 \text { then } \sigma_{t}=0 \text { and }\left|\frac{\tilde{y}_{t, \hat{\theta}_{t}^{u s}}}{\delta_{t}}\right| \leq 1
\end{array}\right.
$$

$\delta_{t}$ is then a bound on the magnitude of the unstructured a posteriori prediction error. This bound has to be specified taking into account the bound $\delta_{v_{t}}$.

\section{about stage 2:}

The second part of the algorithm as to realize the structuring of $\hat{\theta}_{t}^{u s}$. This is done by a function $S():. \hat{\theta}_{t}^{s}=S\left(\hat{\theta}_{t}^{u s}\right)$. This function will be detailed in subsection III-C (see table I page 5).

The interest of matrix $W_{t}$ is to maintain the stability of the algorithm. In fact, the use of that structuring step introduce a correction term $w_{t}$ in the following manner

$$
w_{t}=\hat{\theta}_{t}^{s}-\hat{\theta}_{t}^{u s}
$$

and that correction term disturbs the algorithm behavior. So as to take into account this disturbance $W_{t}$ is designed in the following manner:

$$
W_{t}:\left\{\begin{array}{l}
W_{t}=0 \text { if } w_{t}=0 \\
W_{t} \text { such that } w_{t}^{T} W_{t}^{-1} w_{t} \leq 1 \text { otherwise }
\end{array}\right.
$$

$0<\zeta<1$ is a design parameter whose role will be discussed later.

Let notice that without the second stage, the above algorithm is similar to OBE algorithms presented in [6], [25] and [5]. These algorithms can be apply to any processes satisfying $y_{t}=\phi_{t}^{T} \theta+v_{t}$ with $\left|v_{t}\right| \leq \delta_{v_{t}}$. The main interest of the proposed algorithm is the introduction of the correction term $w_{t}$ in the second stage. In this paper this correction term corresponds to a structuring procedure of the estimated parameters vector. It is possible to imagine an other identification problem in which the correction term will have a different role. In the next subsection it is shown that stability and convergence properties can be demonstrated even in presence of that correction term.

\section{B. Stability and convergence properties.}

The analysis of the estimation algorithm is performed via the analysis of the estimation error $\tilde{\theta}_{t}^{s}$ defined by:

$$
\tilde{\theta}_{t}^{s}=\theta^{*}-\hat{\theta}_{t}^{s}
$$


In the first part of the analysis some geometrical interpretations of the adaptation algorithm are provided. To this end let us define for each time $t$ the observation set $\mathscr{S}_{t}$ :

$$
\mathscr{S}_{t}=\left\{\theta \in \mathbb{R}^{n},\left|\frac{y_{t}-\phi_{t}^{T} \theta}{\delta_{t}}\right| \leq 1\right\}
$$

Given $y_{t}$ and $\phi_{t}, \mathscr{S}_{t}$ is the set of all possible $\theta$ which are consistent with the chosen threshold $\delta_{t}$. An important property of that observation set is given in the following propertie.

Propertie 1: Assume that $\delta_{t} \geq \delta_{v_{t}}$, then

$$
\theta^{*} \in \mathscr{S}_{t}
$$

$\mathscr{S}_{t}$ is then a strip in parameter space bounded by two parallel hyperplanes and which contains $\theta^{*}$. The principe of OBE type algorithms is to find a parameters vector included in $\bigcap_{i}^{t} \mathscr{S}_{i}$.

Let define the un-structured ellipsoid $\mathscr{E}_{t}$ :

$$
\mathscr{E}_{t}^{u s}=\left\{\theta \in \mathbb{R}^{n},\left(\theta-\hat{\theta}_{t}^{u s}\right)^{T} P_{t}^{u s-1}\left(\theta-\hat{\theta}_{t}^{u s}\right) \leq 1\right\}
$$

and the structured ellipsoid $\mathscr{E}_{t}$ :

$$
\mathscr{E}_{t}^{s}=\left\{\theta \in \mathbb{R}^{n},\left(\theta-\hat{\theta}_{t}^{s}\right)^{T} P_{t}^{s-1}\left(\theta-\hat{\theta}_{t}^{s}\right) \leq 1\right\}
$$

The following theorem shows that the proposed identification algorithm provides rules for computing $\hat{\theta}_{t}^{s}$ and $P_{t}^{s}$ so that $\mathscr{E}_{t}$ contains the intersection $\left(\mathscr{S}_{t} \cap \mathscr{E}_{t-1}\right)$ (Fig. 3).

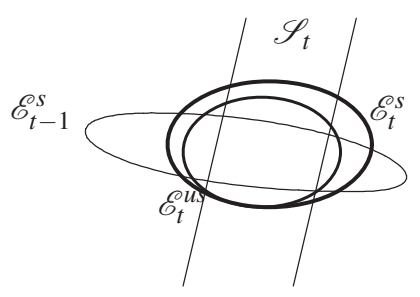

Fig. 3. $\left(\mathscr{S}_{t} \cap \mathscr{E}_{t-1}^{s}\right) \subset \mathscr{E}_{t}^{\text {us }} \subset \mathscr{E}_{t}$

Theorem 1: Consider the class of systems defined in section II and the identification algorithm given by (8), (9), (10) and (12). If $\delta_{t} \geq \delta_{v_{t}}$ hold and suppose that

$$
\theta^{*} \in \mathscr{E}_{t-1}^{s}
$$

then for all initial conditions

- a- An outer bounding ellipsoid of $\left(\mathscr{S}_{t} \cap \mathscr{E}_{t-1}\right)$ is given by $\mathscr{E}_{t}$

- b-

$$
\theta^{*} \in \mathscr{E}_{t}
$$

If furthermore, $\left\{\phi_{t}\right\}$ is a persistently exciting sequence of order $o_{e} \geq n$, i.e there exist $\alpha>0$ and $\beta>0$ such that for all $t \geq o_{e}$

$$
\alpha I_{n} \leq \sum_{i=0}^{o_{e}-1} \phi_{t-i} \sigma_{t-i} \phi_{t-i}^{T} \leq \beta I_{n}
$$

and if there exists $\beta_{W}>0$ such that for all $t$

$$
0 \leq W_{t} \leq \beta_{W} I_{n}
$$

Then the following property holds:

- c- The volume of the ellipsoid $\mathscr{E}_{t}$ is bounded.

A sketch of proof is given in appendix A page 7 .

A consequence of this result is the fact that if in the beginning (i.e. at the time $t=0$ ) $\theta^{*}$ is included in $\mathscr{E}_{0}^{S}$ (this is true with for example $\hat{\theta}_{0}^{s}=0$ and $P_{0}^{s}=\mu I_{n}$ with $\mu$ large enough) then $\theta^{*}$ belongs to $\mathscr{E}_{t}$ for all $t$.

The user parameter $\zeta$ determines the shape of the ellipsoid $\mathscr{E}_{t} s$. Different policies can be considered so as to obtain a particular effect on this ellipsoid (to minimize the product of the length of its axes or to minimize the sum of the squares of the lengths of the axes). It can also be chosen so as to adapt the dynamic of $\hat{\theta}_{t}^{s}$, this will be shown in the next theorem (see (20) and (22)).

The second part of the analysis is performed via the analysis of the estimation error $\tilde{\theta}_{t}^{s}$ which is a non linear function of $\tilde{\theta}_{t-1}^{s}, w_{t}$ and $v_{t}$. Theorem 2 focuses on the behavior of $\left|\tilde{\theta}_{t}^{s}\right|^{2}$. It is shown that under some conditions the center $\hat{\theta}_{t}^{s}$ of the ellipsoid $\mathscr{E}_{t}^{s}$ converges next to the solution $\theta^{*}$.

Theorem 2: Consider the class of systems defined in section II and the identification algorithm given by (8), (9), (10) and (12). If $\delta_{t} \geq \delta_{v_{t}}$ hold and if conditions (18) and (19) in Theorem 1 hold, then for all initial conditions

$$
\begin{aligned}
& \text { - a- for all } t \geq o_{e} \\
& \qquad\left|\tilde{\theta}_{t}^{s}\right|^{2} \leq \gamma_{1}(1-\zeta)^{t} \lambda^{t}\left|\tilde{\theta}_{1}^{s}\right|^{2}+\gamma_{2} \frac{1-(1-\zeta)^{t} \lambda^{t}}{1-(1-\zeta) \lambda}
\end{aligned}
$$

with $\gamma_{1}$ and $\gamma_{2}$ two finite scalars.

If, furthermore, there exists a time $t_{s}$ such that for all $t \geq t_{s}$

$$
w_{t}=0
$$

the following properties hold

- b- for all $t \geq t_{s}+o_{e}$

$$
\left|\tilde{\theta}_{t}^{s}\right|^{2} \leq \gamma_{3}(1-\zeta)^{t-t_{s}} \lambda^{t-t_{s}}
$$

with $\gamma_{3}$ a finite scalar. 
- c- one has

$$
\lim _{t \rightarrow \infty}\left|\frac{\tilde{y}_{t, \hat{\theta}_{t-1}^{s}}}{\delta_{t}}\right| \leq 1
$$

A sketch of proof is given in appendix B page 8 .

Remark 4: Note that if $\delta_{t}=\delta_{v_{t}}$ and $w_{t}=0$ then from (22) we have

$$
\lim _{t \rightarrow \infty} \hat{\theta}_{t}^{s}=\hat{\theta}
$$

where $\hat{\theta}$ parametrizes a model such that:

$$
\begin{aligned}
& \widehat{A}(q) y_{t}=q^{-d} \widehat{B}(q) \widehat{x}_{t}+\tilde{y}_{t} \\
& \widehat{x_{t}}=\widehat{h}\left(u_{t}\right) \\
& \left|\tilde{y}_{t}\right| \leq \delta_{v_{t}}
\end{aligned}
$$

which is coherent with the objective stated in section II.

\section{Design of function $S($.$) .$}

The aim of this subsection is to propose a procedure for the design of a structured estimated parameters vector $\hat{\theta}_{t}^{s}$ from $\hat{\theta}_{t}^{u s}$ in the case where $\hat{\theta}_{t}^{u s}$ is not structured as (7). Such procedure corresponds to function $S($.$) :$

$$
\hat{\theta}_{t}^{s}=S\left(\hat{\theta}_{t}^{u s}\right)
$$

This function $S($.$) must satisfy two conditions:$

- The correction term $w_{t}=\hat{\theta}_{t}^{s}-\hat{\theta}_{t}^{u s}$ must be such that

$$
W_{t}:\left\{\begin{array}{l}
W_{t}=0 \text { if } w_{t}=0 \\
W_{t} \text { such that } w_{t}^{T} W_{t}^{-1} w_{t} \leq 1 \text { otherwise }
\end{array}\right.
$$

The existence of a scalar $\beta_{W}$ such that $W_{t} \leq \beta_{W} I_{n}$ is needed in Theorem 1 and Theorem 2.

- $\hat{\theta}_{t}^{s}$ must satisfy $\left|\frac{\tilde{y}_{t, \hat{\theta}_{t}^{s}}}{\delta_{t}}\right| \leq 1$.

The proposed function $S($.$) corresponds to the 5$ steps procedure given in table I.

The interest of this procedure is twofold:

- It provides structuring of $\hat{\theta}_{t}^{s}$.

- $\hat{\theta}_{t}^{s}$ is such that the magnitude of the a posteriori prediction error $\tilde{y}_{t, \hat{\theta}_{t}^{s}}$ is bounded by $\delta_{t}$.

The condition on the existence of a finite scalar $\beta_{W}$ is difficult to guarantee in a such recursive algorithm. The procedure of table I is ill-conditioned if the parameters $\left\{\mu_{i}\right\}$ extracted in step 1 are close to zero. This is the reason why we advice the use of the above procedure and the use of the second stage of the algorithm after a few times. Typically the implementation of the procedure must be realized only after $n_{a}+n_{b}+n_{h}$ samples (this corresponds to the number of parameters to estimate). Numerous simulations have shown the efficiency of that procedure. This is illustrated in the next section.
Function $S($.$) - Estimation of a structured parameters vector$ step 1:Extract the parameters $\left\{a_{i}\right\}$ and $\left\{\mu_{i}\right\}$ from $\hat{\theta}_{t}^{u s}$.

step 2: If $\sum_{i=1}^{n_{h}} \mu_{i}^{2} \neq 0$, compute a first estimation of $P=$ $\left(\begin{array}{c}b_{1} \\ \vdots \\ b_{n_{b}}\end{array}\right)$ from $\hat{\theta}_{t}^{u s}$. This estimation is denoted
$P^{(1)}$. $\sum_{i=1}^{n_{h}} \mu_{i}^{2}=0$, take $P^{(1)}=0$. step 3: Compute the predictor $\hat{y}_{t}=\varphi_{t}^{T} P^{(1)}+z_{t}$. step 4: Realize a second estimation $P^{(2)}$ as follows:

$$
P^{(2)}=P^{(1)}+\frac{\varphi_{t} \sigma_{t}^{\prime}}{1+\varphi_{t}^{T} \varphi_{t} \sigma_{t}^{\prime}}\left(y_{t}-\hat{y}_{t}\right)
$$

with

$$
\sigma_{t}^{\prime}=\left\{\begin{array}{c}
\frac{1}{\varphi_{t}^{T} \varphi_{t}}\left(\left|\frac{y_{t}-\hat{y}_{t}}{\delta_{t}}\right|-1\right) \\
\text { if }\left(\left|\frac{y_{t}-\hat{y}_{t}}{\delta_{t}}\right|>1\right) \text { and }\left(\varphi_{t}^{T} \varphi_{t}>0\right) \\
0 \text { otherwise }
\end{array}\right.
$$

step 5:Finally $\hat{\theta}_{t}^{s}$ is the parameterized vector built from the estimates for $\left\{a_{i}\right\}$ (step 1), $\left\{b_{i}\right\}$ (step 4) and $\left\{\mu_{i}\right\}$ (step 1).

TABLE I

Remark 5: It is possible to make use of a Singular Values Decomposition (SVD) in step 2. The algorithm will have a higher computational complexity, however it will be numerically more robust when dealing with ill-conditioned data.

\section{NUMERICAL EXAMPLE}

Numerical data have been generated according to (1), (2), (3) and (4). The following system has been considered in [4]:

$$
\begin{gathered}
\left\{\begin{array}{l}
q^{-d} B(q)=q^{-1}\left(1+0.5 q^{-1}+0.5 q^{-2}\right) \\
A(q)=1-0.3 q^{-1}-0.2 q^{-2}+0.3 q^{-3}
\end{array}\right. \\
h\left(u_{t}\right)=0.9545 h_{1}\left(u_{t}\right)+0.2983 h_{2}\left(u_{t}\right)
\end{gathered}
$$

with $h_{1}\left(u_{t}\right)=u_{t}$ and $h_{2}\left(u_{t}\right)=2 u_{t}^{2}$.

The input $u_{t}$ is white noise uniformly distributed in $[-1 ; 1]$ of length $N=4000 . v_{t}$ is a noise generated in the following manner:

$$
v_{t}=\delta_{v_{t}} \frac{1}{2}\left(e_{t}+r_{t}\right)
$$

where $r_{t}$ is a binary square signal with a period of 20 samples, $e_{t}$ is a white noise uniformly distributed in $[-1 ; 1]$ and $\delta_{v_{t}}$ is close to 0.7 in order to have a signal to noise ratio close to $\mathrm{SNR}=5 \mathrm{~dB}$.

The parameters $\left\{a_{i}\right\},\left\{b_{i}\right\}$ and $\left\{\mu_{i}\right\}$ are estimated with three procedures:

- Procedure 1: this first procedure corresponds to the identification of an extended ARX structure using (6) 


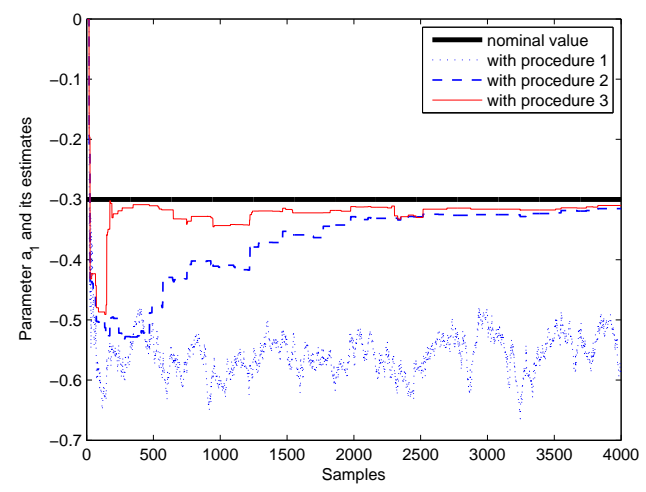

Fig. 4. Simulation results component $a_{1}$

and the ordinary least square algorithm. In order to separate the parameters $\left\{b_{i}\right\}$ and $\left\{\mu_{i}\right\}$ a SVD is applied at each iterations.

- Procedure 2: this second procedure corresponds to our identification algorithm without stage 2. However the parameters $\left\{b_{i}\right\}$ and $\left\{\mu_{i}\right\}$ are separated using the procedure presented in subsection III-C. In fact this amounts to not use the structured parameters vector estimated at time $t$ for time $t+1$. The threshold $\delta_{t}$ is taken equal to $\delta_{v_{t}}$.

- Procedure 3: this third identification procedure is our identification algorithm using stages 1 and 2. Here again $\delta_{t}=\delta_{v_{t}}$

In Fig. 4, 5 and 6 the thick solid lines denote the true parameters and the other lines the parameters estimates. These figures present the estimations for $a_{1}, b_{1}$ and $\mu_{1}$. The other components have similar behavior. In Fig. 7, 8 and 9 the solid lines denote thresholds $\pm \delta_{v_{t}}$, dotted lines correspond to the a posteriori prediction error for each identification procedure.

From these figures we conclude the following:

- Procedure 1 is the only procedure for which the a posteriori prediction error is not bound by $\delta_{t}$.

- Procedure 1 clearly delivers biased estimates (see Fig. 4 and 5). This is due to the fact that the disturbing term $v_{t}$ is not a white noise, the ordinary least square algorithm is not adapted to the considered identification problem.

- The estimations computed with procedures 2 and 3 converge towards a neighborhood of the true parameters. The difference between procedures 2 and 3 lies in the introduction of the correction term $w_{t}$ in procedure 3 . This correction term affects the behavior of the algorithm without loss of the stability property as demonstrated in subsection III-B.

- An interesting point is the fact that the convergence towards the true parameters is faster with procedure 3 . This is a consequence of taking into account the specific structure of the parameters vector i.e. the use of stage 2 (here again the effect $w_{t}$ and $W_{t}$ ).

\section{CONCLUSION AND FUTURE WORKS}

A recursive algorithm for Hammerstein systems identification has been proposed. This algorithm is adapted for

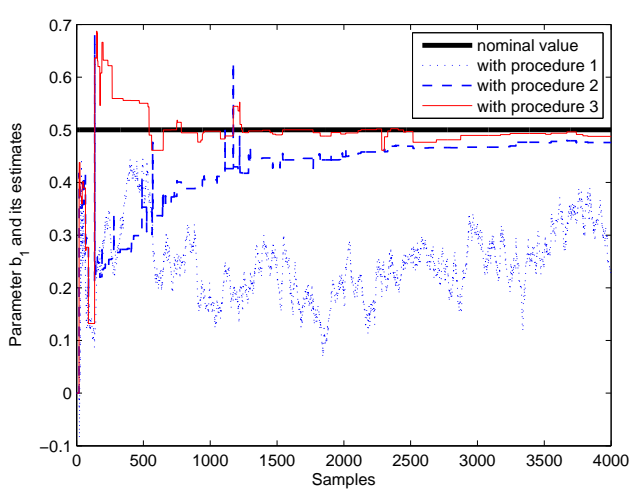

Fig. 5. Simulation results component $b_{1}$

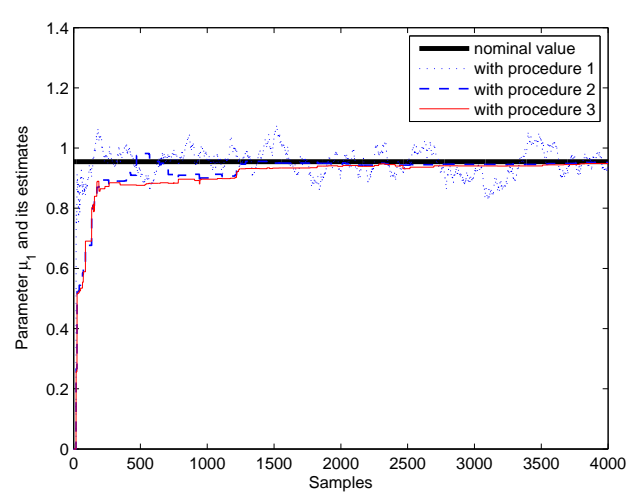

Fig. 6. Simulation results component $\mu_{1}$

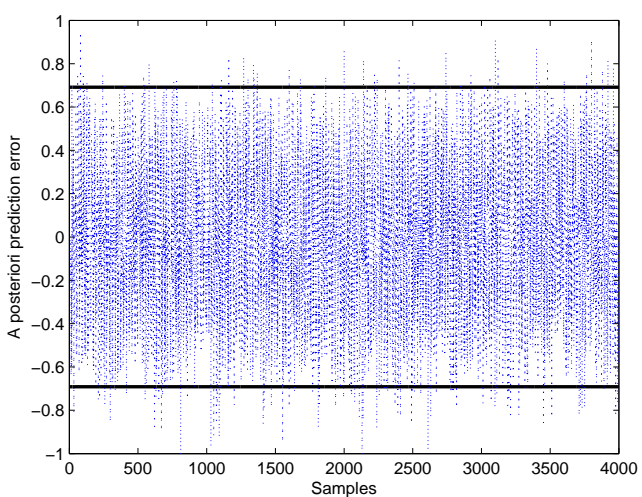

Fig. 7. A posteriori prediction error and threshold $\pm \delta_{v_{t}}$ with procedure 1

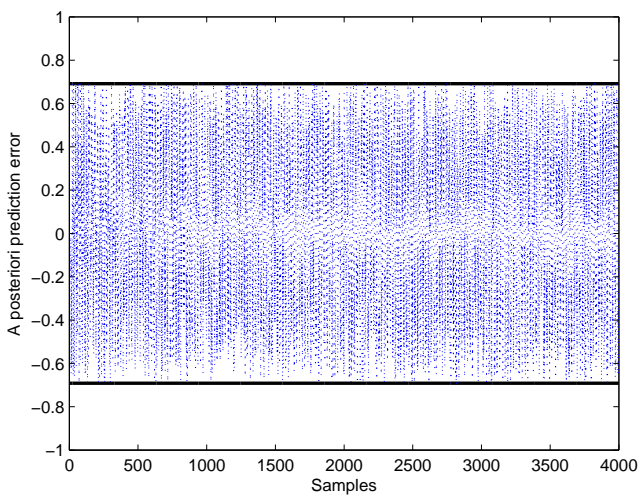

Fig. 8. A posteriori prediction error and threshold $\pm \delta_{v_{t}}$ with procedure 2 


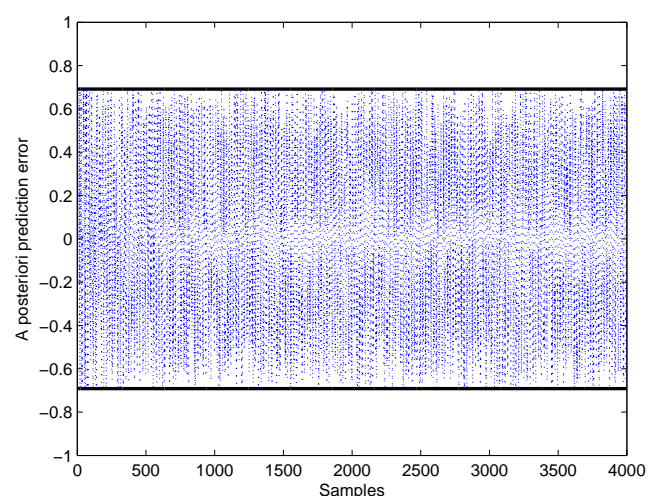

Fig. 9. A posteriori prediction error and threshold $\pm \delta_{v_{t}}$ with procedure 3

the bounded noise case. It consists in two successive stages: the first stage realizes an update of the estimate from new measurements, the second stage allows an online separation of the nonlinear part and the linear part. Stability and convergence properties have been investigated. The second stage of the algorithm consists in the introduction of a "disturbance term" on the parameters computed in the first stage. This "disturbance term" is used here in order to separate the nonlinear part and the linear part, however it could be used so as to impose other constraints on the parameters (impose some parameters to be positive for example). Future researches will concentrate on the use of that two stages algorithm on some other applications. In this paper the linear part is an ARX process, we want also to generalize our results on systems such that the linear part is parametrized by an $\mathrm{OE}$ process.

\section{REFERENCES}

[1] A. Vicino A. Garulli and G. Zappa. Conditional central algorithms for worst-case set membership indentification and filtering. IEEE Transactions on automatic control, 45(1):1423, 2000.

[2] E. Bai. An optimal two-stage identification algorithm for hammersteinwiener nonlinear systems. Automatica, 34(3):333 - 338, 1998.

[3] E. Bai. Identification of linear systems with hard input nonlinearities of known structure. Automatica, 38(5):853 - 860, 2002.

[4] E. Bai and K. Li. Convergence of the iterative algorithm for a general hammerstein system identification. Automatica, 46(11):1891 - 1896, 2010.

[5] M. Boutayeb, Y. Becis, and M. Darouach. Recursive identification of linear multivariable systems with bounded disturbances. Proceedings $15^{\text {th }}$ IFAC World Congress-Barcelona, 2002.

[6] C. Canudas-De-Wit and J. Carrillo. A modified EW-RLS algorithm for systems with bounded noise. Automatica, 26(3):599 - 606, 1990.

[7] V. Cerone and D. Regruto. Parameter bounds for discrete-time hammerstein models with bounded errors. IEEE Transactions on Automatic Control, 48(10):1855-1860, 2003.

[8] F. Chang and R. Luus. A noniterative method for identification using hammerstein models. IEEE Transactions on Automatic Control, 16(5):464468, 1971.

[9] S. Dasgupta and Y-F Huang. Asymptotically convergent modified recursive least square with data-dependent updating and forgetting factor for systems with bounded noise. IEEE Transactions on information theory, 33(3):383-392, 1987.

[10] F. Ding and T. Chen. Identification of hammerstein nonlinear armax systems. Automatica, 41(9):1479 - 1489, 2005.

[11] E. Fogel and Y.F. Huang. On the value of informaton in system identification - bounded noise case. Automatica, 18(2):229-238, 1982.

[12] A. Garulli, B.Z. Kacewicz, A. Vicino, and G. Zappa. Error bounds for conditional algorithms in restricted complexity set membership identification. IEEE Transactions on automatic control, 45(1):160 $164,2000$.
[13] F. Giri and E. Bai. Block Oriented Nonlinear System Identification. Springer-Verlag, 2010.

[14] F. Giri, Y. Rochdi, F.Z. Chaoui, and A. Brouri. Identification of hammerstein systems in presence of hysteresis-backlash and hysteresisrelay nonlinearities. Automatica, 44(3):767-775, 2008.

[15] W. Greblicki and M. Pawlak. Identification of discrete hammerstein systems using kernel regression estimates. IEEE Transactions on Automatic Control, 31(1):74 - 77, 1986.

[16] N.D. Haist, F. Chang, and R. Luus. Nonlinear identification in the presence of correlated noise using a hammerstein model. IEEE Transactions on Automatic Control, 18(5):552 - 555, 1973.

[17] Y. Han and R.D. De Callafon. Hammerstein system identification using nuclear norm minimization. Automatica, 48(9):2189 - 2193, 2012.

[18] F. Le, I. Markovsky, C. Freeman, and E. Rogers. Recursive identification of hammerstein systems with application to electrically stimulated muscle. Control Engineering Practice, 20:386 - 396, 2012.

[19] L. Ljung. System identification: theory for the user. Prentice Hall, 1999.

[20] R. Lozano-Leal and R. Ortega. Reformulation of the parameter identification problem for systems with bounded disturbances. Automatica, 23(2):247-251, 1987.

[21] M. Milanese and G. Belforte. Estimaton theory and uncertainty intervals evaluation in presence of unknown but bounded errors. linear families of models and estimators. IEEE Transactions on automatic control, 27(2):408-414, 1982.

[22] K. Narendra and P. Gallman. An iterative method for the identification of nonlinear systems using a hammerstein model. IEEE Transactions on Automatic Control, 11(3):546 - 550, 1966.

[23] F.C. Schweppe. Uncertain dynamic systems. Prentice-Hall, 1973.

[24] X-F. Sun and Y-Z. Fan. Comments on "identification for systems with bounded noise". IEEE Transactions on automatic control, 46(5):808809, 2001.

[25] G. Tan, C. Wen, and Y.C. Soh. Identification for systems with bounded noise. IEEE Transactions on automatic control, 42(7):998-1001, 1997.

[26] A. Vicino and G. Zappa. Sequential approximation of parameter sets for identification with parametric and nonparametric uncertainty. $32^{\text {th }}$ IEEE Control and Decision Conference-New York, 1993.

[27] W. Zhao and H. Chen. Recursive identification for hammerstein system with arx system. IEEE Transactions on Automatic Control, 51(12):1966-1974, 2006.

[28] W. Zhao and H. Chen. Adaptive tracking and recursive identification for hammerstein systems. Automatica, 45(12):2773-2783, 2009.

\section{A- Proof of Theorem 1}

For lack of space we do not detail all the proof, we will simply state the essential points.

- Sketch of proof for $\mathscr{S}_{t} \cap \mathscr{E}_{t-1} \subset \mathscr{E}_{t}$ us.

Consider $\theta$ such that $\theta \in \mathscr{E}_{t-1}$ and $\theta \in \mathscr{S}_{t}$. From (13) and (15) this gives

$$
\begin{gathered}
\lambda\left(\theta-\hat{\theta}_{t-1}^{s}\right)^{T} P_{t-1}^{s-1}\left(\theta-\hat{\theta}_{t-1}^{s}\right)+\left(y_{t}-\phi_{t}^{T} \theta\right)^{2} \sigma_{t} \\
\leq \lambda+\tilde{y}_{t, \hat{\theta}_{t-1}^{s}}^{2} \sigma_{t}\left(\frac{\delta_{t}}{\tilde{y}_{t, \hat{\theta}_{t-1}^{s}}^{s}}\right)^{2}
\end{gathered}
$$

This can be rewritten as:

$$
\begin{gathered}
\left(\theta-\hat{\theta}_{t}^{s}\right)^{T} P_{t}^{s-1}\left(\theta-\hat{\theta}_{t}^{s}\right) \\
\leq \lambda+\tilde{y}_{t, \hat{\theta}_{t-1}^{s}}^{2}\left(\sigma_{t}\left(\frac{\delta_{t}}{\tilde{y}_{t, \hat{\theta}_{t-1}^{s}}^{s}}\right)^{2}-\frac{\lambda \sigma_{t}}{\lambda+\phi_{t}^{T} P_{t-1}^{s} \phi_{t} \sigma_{t}}\right)
\end{gathered}
$$

If $\sigma_{t}=0$ then $\left(\theta-\hat{\theta}_{t}^{s}\right)^{T} P_{t}^{s-1}\left(\theta-\hat{\theta}_{t}^{s}\right)=\lambda$ and if $\sigma_{t} \neq 0$ it can shown that $\left(\sigma_{t}\left(\frac{\delta_{t}}{\tilde{y}_{t, \hat{\theta}_{t-1}^{s}}^{s}}\right)^{2}-\frac{\lambda \sigma_{t}}{\lambda+\phi_{t}^{T} P_{t-1}^{s} \phi_{t} \sigma_{t}}\right)<0$ thus we always get

$$
\left(\theta-\hat{\theta}_{t}^{s}\right)^{T} P_{t}^{s-1}\left(\theta-\hat{\theta}_{t}^{s}\right) \leq \lambda
$$


Together with $0<\lambda \leq 1$, this gives

$$
\left(\theta-\hat{\theta}_{t}^{s}\right)^{T} P_{t}^{s-1}\left(\theta-\hat{\theta}_{t}^{s}\right) \leq \lambda
$$

and then $\theta \in \mathscr{E}_{t} u s$. We conclude that $\mathscr{S}_{t} \cap \mathscr{E}_{t-1} \subset \mathscr{E}_{t} u s$.

- Sketch of proof for $\mathscr{S}_{t} \cap \mathscr{E}_{t-1} \subset \mathscr{E}_{t}$ us .

This result is based on the following lemma from [23]: Lemma 1: [23] Consider two ellipsoids $\mathscr{E}_{i}=$ $\left\{\theta ;\left(\theta-\theta_{c i}\right)^{T} P_{i}^{-1}\left(\theta-\theta_{c i}\right) \leq 1\right\}$ and their sum $\mathscr{E}_{1} \oplus \mathscr{E}_{2}=\left\{\theta=\theta_{1}+\theta_{2} ; \theta_{1} \in \mathscr{E}_{1}\right.$ and $\left.\theta_{2} \in \mathscr{E}_{2}\right\}$. Then $\forall \zeta$ such that $0<\zeta<1$, we have $\mathscr{E}_{3} \supseteq \mathscr{E}_{1} \oplus \mathscr{E}_{2}$ with $\mathscr{E}_{3}=\left\{\theta ;\left(\theta-\theta_{c 3}\right)^{T} P_{3}^{-1}\left(\theta-\theta_{c 3}\right) \leq 1\right\}, \theta_{c 3}=\theta_{c 1}+\theta_{c 2}$ and $P_{3}=\frac{P_{1}}{1-\zeta}+\frac{P_{2}}{\zeta}$.

Here we take $P_{1}=P_{t}^{u s}, P_{2}=W_{t}$ and $P_{3}=P_{t}^{s}$ and $\theta_{c 3}=$ $\theta_{c 1}+\theta_{c 2}$ corresponds to $\hat{\theta}_{t}^{s}=\hat{\theta}_{t}^{u s}+w_{t}$.

- From the two previous results it is easy to conclude $\mathscr{S}_{t} \cap \mathscr{E}_{t-1} \subset \mathscr{E}_{t}^{\text {us }} \subset \mathscr{E}_{t}$, this corresponds to proposition $a-$.

Using assumption (16) and the fact that $\delta_{t} \geq \delta_{v_{t}}$, proposition $b-$ is a consequence of proposition $a-$.

- Sketch of proof that there exists a scalar $s_{\text {sup }}$ such that $P_{t}^{s-1} \leq s_{\text {sup }} I_{n}$.

From (9) we have $P_{t}^{s}=\frac{1}{1-\zeta} P_{t}^{u s}+\frac{1}{\zeta} W_{t}$ and $W_{t} \geq 0$, thus $P_{t}^{s} \geq \frac{1}{1-\zeta} P_{t}^{u s}$ From (8) this gives

$$
\begin{gathered}
P_{t}^{s-1} \leq(1-\zeta) \lambda P_{t-1}^{s-1}+(1-\zeta) \phi_{t} \sigma_{t} \phi_{t}^{T} \\
P_{t}^{s-1} \leq(1-\zeta)^{t} \lambda^{t} P_{1}^{s-1}+(1-\zeta) \sum_{i=0}^{t-1}(1-\zeta)^{i} \lambda^{i} \phi_{t-i} \sigma_{t-i} \phi_{t-i}^{T}
\end{gathered}
$$

We have $0<\zeta<1$ and $0<\lambda \leq 1$, consequently there exist $s_{\text {sup }}$ such that $P_{t}^{s-1} \leq s_{\text {sup }} I_{n}$.

- Sketch of proof that there exists a scalar $s_{\text {inf }} \neq 0$ such that $P_{t}^{s-1} \geq s_{\text {inf }} I_{n}$.

From (9) we have

$$
P_{t}^{s}=\frac{1}{1-\zeta} P_{t}^{u s}\left(I_{n}+\frac{1-\zeta}{\zeta} P_{t}^{u s-1} W_{t}\right)
$$

Let us denote $J_{t}=I_{n}+\frac{1-\zeta}{\zeta} P_{t}^{u s-1} W_{t}$, this gives together with (8)

$$
P_{t}^{s-1}=(1-\zeta) \lambda J_{t}^{-1} \lambda P_{t-1}^{s-1}+(1-\zeta) J_{t}^{-1} \phi_{t} \sigma_{t} \phi_{t}^{T}
$$

After $o_{e}$ iterations we get

$$
\begin{gathered}
P_{t}^{s-1}=(1-\zeta)^{o_{e}} \lambda^{o_{e}} \prod_{i=0}^{o_{e}-1} J_{t-i}^{-1} \lambda P_{t-o_{e}}^{s-1}+ \\
\sum_{i=0}^{o_{e}-1}(1-\zeta)^{i} \lambda^{i} \prod_{k=0}^{i} J_{t-k}^{-1}(1-\zeta) \lambda \phi_{t-i} \sigma_{t-i} \phi_{t-i}^{T}
\end{gathered}
$$

From (26) and (19) $P_{t}^{s-1} W_{t} \leq s_{\text {sup }} \beta_{W} I_{n}$, then there exists a scalar $s_{J}$ such that $I_{n} \geq J_{t}^{-1} \geq s_{J}^{-1} I_{n}$. Together with (27) we show

$$
P_{t}^{s-1} \geq(1-\zeta) \sum_{i=0}^{o_{e}-1}(1-\zeta) \lambda^{i} s_{J}^{-i} \phi_{t-i} \sigma_{t-i} \phi_{t-i}^{T}
$$

It can be shown that there exists a scalar $\eta$ such that

$$
P_{t}^{s-1} \geq \eta \sum_{i=0}^{o_{e}-1} \phi_{t-i} \sigma_{t-i} \phi_{t-i}^{T}
$$

together with condition (18) we get $P_{t}^{s-1} \geq \eta \alpha I_{n} \neq 0$, then there exists a scalar $s_{\text {inf }} \neq 0$ such that $P_{t}^{s-1} \geq$ $s_{\text {inf }} I_{n}$.

- Proposition $c-$ is a consequence of the fact that $P_{t}^{s}$ is bounded.

\section{B- Proof of Theorem 2}

For lack of space we do not detail all the proof, we will simply state the essential points. Consider the following Lyapounov function:

$$
V_{t}^{s}=\tilde{\theta}_{t}^{s T} P_{t}^{-1} \tilde{\theta}_{t}^{s}
$$

with $\tilde{\theta}_{t}^{s}=\theta^{*}-\hat{\theta}_{t}^{s}$.

- Sketch of proof on the relation between $V_{t}^{s}$ and $V_{t}^{u s}$. $V_{t}^{s}$ can be rewritten as

$$
V_{t}^{s}=\left(\tilde{\theta}_{t}^{u s}-w_{t}\right)^{T}\left(\frac{1}{1-\zeta} P_{t}^{u s}+\frac{1}{\zeta} W_{t}\right)^{-1}\left(\tilde{\theta}_{t}^{u s}-w_{t}\right)
$$

We have $\tilde{\theta}_{t}^{u s}{ }^{T} P_{t}^{u s}{ }^{-1} \tilde{\theta}_{t}^{u s} \leq 1$ and $w_{t}^{T} W_{t}^{-1} w_{t} \leq 1$ this gives

$$
V_{t}^{s} \leq(1-\zeta) V_{t}^{u s}+\zeta w_{t}^{T} W_{t}^{-1} w_{t}
$$

- Sketch of proof on the relation between $V_{t}^{u s}$ and $V_{t-1}^{s}$. Using the fact that $\left\|\tilde{y}_{t, \hat{\theta}_{t}^{u s}}\right\| \leq \delta_{t}$ and $\delta_{v_{t}} \leq \delta_{t}$ it can be shown that

$$
V_{t}^{u s} \leq \lambda V_{t-1}^{s}
$$

- Sketch of proof proposition a-

From (28) and (29) we obtain

$$
V_{t}^{s} \leq(1-\zeta)^{t} \lambda^{t} V_{1}^{s}+\zeta \sum_{i=0}^{t-1}(1-\zeta)^{i} \lambda^{i}
$$

We have shown that $0<s_{\text {inf }} I_{n} \leq P_{t}^{s-1} \leq s_{\text {sup }} I_{n}$, then

$$
s_{\text {inf }}\left|\tilde{\theta}_{t}^{s}\right|^{2} \leq(1-\zeta)^{t} \lambda^{t} V_{1}^{s}+\zeta \sum_{i=0}^{t-1}(1-\zeta)^{i} \lambda^{i}
$$

Proposition a- is a consequence of this inequality with $\gamma_{1}=\frac{\mu_{\max }\left(P_{1}^{s-1}\right)}{s_{\text {inf }}}$ and $\gamma_{2}=\frac{\zeta}{s_{\text {inf }}}$.

- Sketch of proof proposition b-

If there exists a time $t_{s}$ such that for all $t \geq t_{s} w_{t}=0$, it can be shown that

$$
V_{t}^{s} \leq(1-\zeta)^{t-t_{s}} \lambda^{t-t_{s}} V_{t-t_{s}}^{s}
$$

then with $t \geq t_{s}+o_{e}$

$$
s_{\text {inf }}\left|\tilde{\theta}_{t}^{s}\right|^{2} \leq(1-\zeta)^{t-t_{s}} \lambda^{t-t_{s}} V_{t-t_{s}}^{s}
$$

Proposition $\mathrm{b}$ - is a consequence of this inequality with $\gamma_{3}=\frac{V_{t-t_{s}}^{s}}{s_{\text {inf }}}$. With $0<\lambda \leq 1$ and $0<\zeta<1$, proposition c- follows from proposition $b-$. 